\title{
Distributed Parameters Dynamic Model of a Solar Fresnel Collector Field ${ }^{\star}$
}

\author{
Marco Spoladore* Eduardo F. Camacho* M. Elena Valcher** \\ * System and Automation Engineering Department, University of \\ Seville, Spain (e-mail: spola20@libero.it, eduardo@esi.us.es) \\ ** Department of Information Engineering, University of Padova, Italy \\ (e-mail:meme@dei.unipd.it)
}

\begin{abstract}
This paper describes a dynamic model for a linear Fresnel collector field of a solar refrigeration plant. The collector field concentrates solar radiations on a tube that heats up water that is used by an absorption machine to produce chilled air for refrigeration purposes. The model takes into account the solar radiation losses produced by the mirrors and the absorbing tube structure as well as the temperatures in 64 segments of the receiving tube and water. Although the dynamic model is of high dimension and nonlinear, the identification problem can be expressed as a linear problem in model parameters. The least square method was used for identification. The model was validated comparing the simulation results with the plant data for different operating conditions.
\end{abstract}

\section{INTRODUCTION}

In today's climate of growing energy needs and increasing environmental concerns, alternatives to the use of nonrenewable and polluting fossil fuels need to be searched for. One such alternative is Solar Energy. The sun creates its energy through a thermonuclear process that creates heat and electromagnetic radiation. The heat remains in the sun and is instrumental in maintaining the thermonuclear reaction. The electromagnetic radiation streams out into space in all directions. Only a very small fraction of the total radiation reaches the Earth, where it is collected.

Solar energy is radiated from the sun through forms of heat and light, and all Solar Technology Systems capture the energy of the sun by absorbing light as heat. There are two main types of solar technologies: Thermosolar technology and Photovoltaic technology. Thermosolar Power Systems concentrate sunlight, usually with mirrors, to heat to high temperatures a fluid that drives an engine. This approach differs from the one used by Photovoltaic Solar Power Systems, where light interacts directly with special materials to separate charges and generate electricity. The world of Solar technologies consists of 4 major areas: (1) Concentrating Solar Power Systems; (2) Photovoltaic Systems; (3) Solar Heating Systems and (4) Solar Lighting Systems.

This paper focus on Concentrating Solar Power Systems, in particular linear concentrator systems in which reflective materials focus the sun's heat energy that then drives a generator to produce electricity. Specifically, we will consider the Linear Fresnel Reflector System of the Seville University's Thermosolar Plant, that will be described in the next section. The paper provides a dynamic model for this system whose main purpose is to help developing a

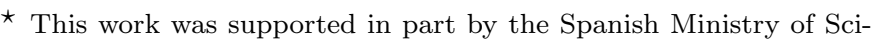
ence and Innovation, Junta de Andalucia and Gas Natural SDG S.A. under grants DPI2008-05818, P07-TEP-02720 and PI-0709/2009
}

control scheme for this type of plants. Fresnel concentrators based on the approximation of the optical behavior by ray-tracing have been developed (Larbi et al. [2000], Facao et al. [2009]). These non-dynamical models were developed to evaluate and optimize collectors design. This paper presents a dynamic model of the Fresnel collector field that allows to simulate optical factors such as the shades produced by the facets, the tube structure, the temperature of the metal tube and of the heated water. A dynamic model of parabolic trough collectors has been developed (Camacho et al. [1997]) for the solar platform of Almeria (PSA).

The paper is organized as follows: section 2 describes the Linear Fresnel Collector System and section 3 provides the related optical model. The heat interchange in the receptor pipe is described by the thermal model in section 4 , while model validation results are shown in section 5 .

\section{LINEAR FRESNEL REFLECTOR SYSTEM}

Linear Concentrating Solar Power (CSP) collectors are one of the three types of CSP systems in use today. They include the two major types of linear concentrator systems: Parabolic Trough Systems and Linear Fresnel Reflector Systems. Linear CSP collectors capture the sun's energy with large mirrors that reflect and focus the sunlight onto a linear receiver tube. The receiver contains a fluid that is heated by the sunlight, and then used to create superheated steam that spins a turbine that drives a generator to produce electricity. Alternatively, steam can be generated directly in the solar field, eliminating the need for costly heat exchangers.

Linear concentrating collector fields consist of a large number of collectors in parallel rows that are typically aligned in a north-to-south orientation to maximize both annual and summertime energy collection. With a singleaxis sun-tracking system, this configuration enables the 
mirrors to track the sun from east to west during the day, ensuring that the sun reflects continuously onto the receiver tubes.

In linear Fresnel reflector systems, flat or slightly curved mirrors mounted on trackers on the ground are configured to reflect sunlight onto a receiver tube located above these mirrors. A small parabolic mirror is sometimes added atop the receiver, to further focus the sunlight. In the thermosolar plant situated in Seville's Engineering College, the solar energy collected by a Linear Fresnel collector field is used by an absorption machine to provide cooling for the College.

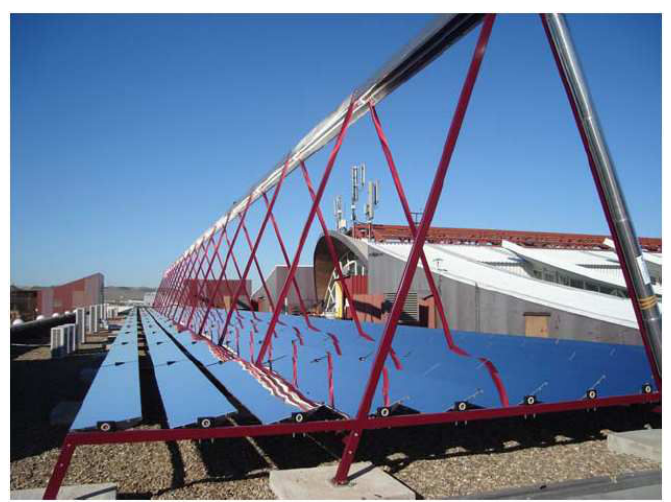

Fig. 1. Seville's Thermosolar Plant

The solar plant functioning can be schematically divided into three phases:

(1) Collector mirrors reflect sunlight to an absorber pipe situated on a plane parallel to that of collector, but located over it.

(2) A heat-transport fluid (water) flows through the pipe, absorbing heat from the walls of the duct to warm up, increasing its own temperature.

(3) The hot fluid enters the absorption machine, that exploits water's heat to refrigerate the buildings of Seville Engineering's College.

The thermosolar part of the plant consists of:

- Fresnel flat collectors. In fact, they are not completely flat, but slightly curved, with a curvature obtained by a process of cold bending.

- A receiver, formed by a single pipe, with covering. The receiver is situated on a plane parallel to flat collectors, over them. Inside the pipe flows the heat transport (or heat-carrier) fluid.

- A receiver carrying structure.

- A solar tracking mechanism. In this concentrator typology, each mirrors row moves independently.

The main advantages of a collector field of this type are: mirrors and tracking system's low cost; almost flat collectors placed next to the ground, so that wind loads are reduced; efficient ground use because collector rows can be placed side by side; easy access to mechanic moving parts and surfaces.

In Table 1 technical data and the principal rating values of the elements that constitute the solar plant are reported.

\begin{tabular}{|c|c|}
\hline Occupied Ground Extension & $480 \mathrm{~m}^{2}$ \\
\hline Total Reflecting Surface & $352 \mathrm{~m}^{2}$ \\
\hline Plant Orientation & East-West \\
\hline Nr. of Receptors (Nr. of pipes) & 1 \\
\hline Receptor's Length & $64 \mathrm{~m}$ \\
\hline Receptor Typology & $\begin{array}{l}\text { Cavity receptor with secondary } \\
\text { reflector and glass cover }\end{array}$ \\
\hline Receptor Plane's Height & $4 \mathrm{~m}$ over collectors plane \\
\hline Receptor width & $0.3 \mathrm{~m}$ \\
\hline Absorber Typology & $\begin{array}{l}\text { Steel pipe DNI 1.4541(AISI 321): } \\
\text { stabilized austenitic stainless steel }\end{array}$ \\
\hline Heat-Transfer Fluid & Water \\
\hline Duct Nominal Pressure & 13 bar \\
\hline Reflecting Mirror Rows Number & 11, each one divided into 2 middle row \\
\hline Single Reflective Mirror Length & $4 \mathrm{~m}$ \\
\hline Single Reflective Mirror Width & $0.5 \mathrm{~m}$ \\
\hline Total Number Of Reflective Mirrors & 176 \\
\hline Mirror Nominal Reflectivity & 0.92 \\
\hline Optical Concentration Ratio & 25 \\
\hline
\end{tabular}

Table 1. Technical data of the solar plant.

\section{OPTICAL MODEL}

The optical model developed has general validity and applies to any solar plant of this typology. In the optical model, reflective mirrors are considered to be flat, because of their high radius of curvature, and the origin of the reference system is placed at the beginning of the mirrors' middle row: the resulting model has the inputs and the outputs indicated in Figure 2. The elaboration of all input data provides all the magnitudes necessary to monitor the plant's functioning and for the thermal model's setting.

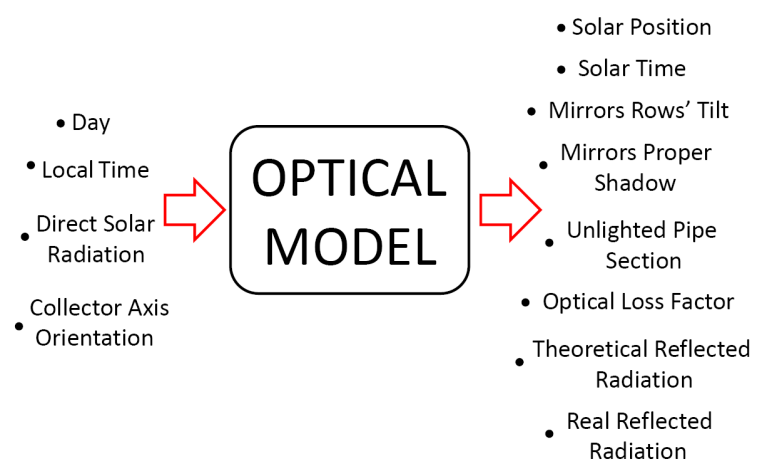

Fig. 2. Optical Model Scheme.

In order to understand the magnitudes of the model, a single mirror is represented in Figure 3, with all the parameters necessary to perform calculations on the model. Referring to Figure 3, ori is the orientation angle between the $\mathrm{x}$ axis and the north-south direction, the vector $\vec{i}$ 
is the solar incidence vector that defines the way the sunrays affect each mirror, and its components depend on the values of solar altitude, azimuth and orientation (see Spoladore [2010] for a more detailed description).

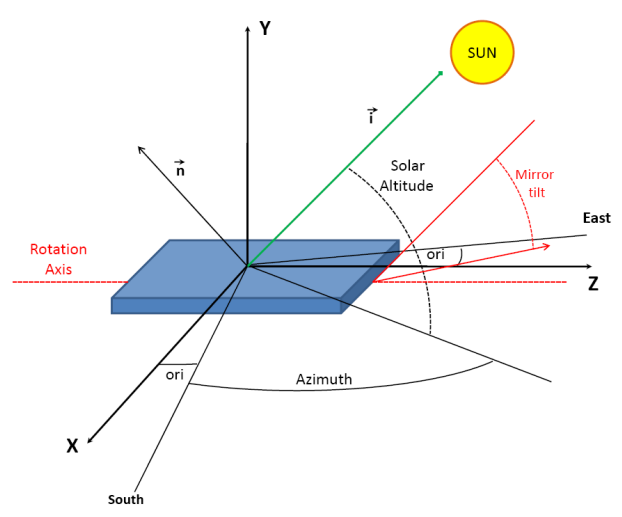

Fig. 3. Reference system applied to a single mirror.

The optical model is obtained by combining a twodimensional model and a three-dimensional one. The 2DModel is developed to calculate the inclination of each mirror row, since each row has the same tilt throughout its length. The model is then used to calculate shadows effects connected with mirrors' tilt and it is the basis for the 3D-Model.

First, the position of each mirror row in an ordinary day at any time has to be calculated. Each row of mirrors has an independent sun-tracking system that rotates along the $\mathrm{z}$ axis, varying its inclination according to the solar altitude, or elevation. The solar incidence vector is projected on the xy-plane to define its position with respect to the mirrors' tilt. The projection is again a vector, $\overrightarrow{i_{2 D}}$, that still depends on the azimuth value $\alpha$. The expression for the two-dimensional vector is the following one, where $\phi$ and $\theta_{s}$ indicate orientation angle and elevation, respectively:

$$
\begin{array}{ll}
\text { (1) } \alpha \geq 0: & \overrightarrow{i_{2 D}}=\left[\begin{array}{c}
\cos \theta_{s} \cdot \cos (\phi+\alpha) \\
\sin \theta_{s}
\end{array}\right] \\
(2) \alpha<0 \text { and }|\alpha| \leq \phi: & \overrightarrow{i_{2 D}}=\left[\begin{array}{c}
\cos \theta_{s} \cdot \cos (\phi-|\alpha|) \\
\sin \theta_{s}
\end{array}\right] \\
\text { (3) } \alpha<0 \text { and }|\alpha|>\phi: & \overrightarrow{i_{2 D}}=\left[\begin{array}{c}
\cos \theta_{s} \cdot \cos (|\alpha|-\phi) \\
\sin \theta_{s}
\end{array}\right]
\end{array}
$$

The vector $\overrightarrow{i_{2 D}}$ represents the sunlight that affects the mirror while $\overrightarrow{r_{2 D}}$ denotes the light reflection on it. Once the incidence vector is determined, the direction of $\overrightarrow{r_{2 D}}$ is forced to be the same as the receptor's, to warm it. Clearly the value of the reflection vector is calculated for each mirror row. Expressing the coordinates of each row as $\left(x_{m}, y_{m}\right)$, where $x_{m}, y_{m}$ are the row positions on $\mathrm{x}$ and $\mathrm{y}$ axis, the reflection vector is obtained through the formula:

$$
\overrightarrow{r_{2 D}}=\left[\begin{array}{c}
r_{2 D_{x}} \\
r_{2 D_{y}}
\end{array}\right]=\left[\begin{array}{c}
\frac{X_{R}-x_{m}}{\sqrt{\left(X_{R}-x_{m}\right)^{2}+\left(Y_{R}-y_{m}\right)^{2}}} \\
\frac{Y_{R}-y_{m}}{\sqrt{\left(X_{R}-x_{m}\right)^{2}+\left(Y_{R}-y_{m}\right)^{2}}}
\end{array}\right]
$$

where $\left(X_{R}, Y_{R}\right)$ are the receptor coordinates. Once the components of $\overrightarrow{r_{2 D}}$ are known, the angle $\beta$, formed by $\overrightarrow{r_{2 D}}$ and the horizontal x-axis, can be calculated as:

$$
\beta=\arctan \left(\frac{r_{2 D_{y}}}{\left|r_{2 D_{x}}\right|}\right) .
$$

The only two magnitudes that are still to be determined are the angles $\alpha$ and $\theta$ : the vector scalar product and laws of reflection can be used to calculate them. Mirrors row inclination can be computed, differentiating the calculation based on the various possible cases.

$$
\begin{array}{ll}
x_{m}<0, & \gamma \geq \beta: \rho=\pi / 2-\beta-\theta \\
x_{m}<0, & \gamma<\beta: \rho=\pi / 2-\beta+\theta \\
x_{m}=0: & \rho=\theta \\
x_{m}>0: & \rho=\beta+\theta-\pi / 2 .
\end{array}
$$

With this procedure, the inclination value of each mirrors row, at every hour of every day, can be determined.

\subsection{Mirrors Shadows}

The 2D-model is utilized also to calculate the total shadows on the collectors surface area. Total shadows that affect mirrors area are the sum of two different components:

- shadows projected by each mirror row on the previous one;

- shadow produced by the receptor on the total collectors area.

For the particular path of the sun above the plant the shadow produced by each mirror can only be from a mirror onto the previous one. The shadow projected by the receptor on the collector's surface is usually smaller in percentage than the mirrors' total shadow and it affects only the negative $\mathrm{x}$ semiaxis. Once the total shadow in the 2D-model has been calculated, the total shadow surface is computed by multiplying the shadow width value along the $\mathrm{x}$ axis by the $\mathrm{z}$ axis length, taking into account the orientation of the $\mathrm{z}$ axis with respect to the south and the mirrors current inclination.

The proper mirror shadow is the sum of the shadows that each mirror projects on the previous one. The possible projected shadow depends not only on the sun elevation, but above all on the corresponding tilt of the adjacent mirror axes. Therefore formulas for proper mirrors shadow calculation are differentiated according to mirror positive or negative inclination. To perform the calculation trigonometric relations are used. Also the receptor shadow, that is the shadow produced by the receptor on the mirror's reflective surface, is calculated using trigonometric relations.

The proper shadow proportion $p_{p s}$ is the ratio between the proper mirrors shaded area and the total collector area $\left(352 \mathrm{~m}^{2}\right)$, while the proper shadow factor $f_{p s}$ is the percentage of mirrors' available reflective area, after 
removing proper mirrors shadow. Clearly, $f_{p s}=1-p_{p s}$. The receptor shadow proportion, $p_{r s}$, and the receptor shadow factor, $f_{r s}$ are similarly defined and in turn are related as $f_{r s}=1-p_{r s}$. Then, sum proper mirrors shadow and receptor shadow, the total shaded zone on the reflective surface is obtained.

$$
p_{\text {tot }}=\frac{p_{p s}+p_{r s}}{352} \quad f_{\text {tot }}=1-p_{\text {tot }}
$$

\subsection{Optical Losses}

The real radiation that reaches the receptor's pipe is not the same one that affects the reflective mirrors, because of optical losses. The incident solar radiation is fully exploited only if its direction is perpendicular to the reflective mirror's surface, but this never happens because mirrors are tilted to reflect solar radiation on the absorber's pipe. Thus, there is always a loss of direct radiation that depends on the angle $\kappa$ that a single mirror forms with the perpendicular to the direction of the solar inclination.

The angle $\kappa$ is determined in two different ways, depending on the mirror's inclination value $\rho$ :

$$
\begin{aligned}
& \rho>0 \rightarrow \kappa=\pi / 2-\gamma-\rho \\
& \rho<0 \rightarrow \kappa=\pi / 2+\rho-\gamma .
\end{aligned}
$$

Optical losses factor of the $i$-th mirrors row is defined as $f_{\text {ol }_{i}}=\cos \kappa_{i}$, where $\kappa_{i}$ is the angle of optical loss relative to $i$-th row. Optical losses factors will be utilized to calculate the real solar radiation that affects receptor's pipe.

Now the 3D-Model is used to calculate the real solar radiation affecting the receptor's pipe. In order to compute this magnitude, the receptor's unlit part has to be ascertained. The unlit part depends on the azimuthal solar component, that influences solar radiation affecting receptor, therefore a 3D-Model has to be utilized. All three components of the solar incidence vector are considered, with the $\mathrm{z}$ component varying between 0 and 64 meters, that is the $\mathrm{z}$ axis length.

The calculation is performed for each mirrors row, and hence 11 different values are obtained. So, the value of unlit part $\delta_{u p_{A}}$ is the average of the 11 values:

$$
\delta_{u p_{A}}=\frac{1}{11} \sum_{i=1}^{11} \delta_{u p_{i}} .
$$

The receptor unlit part factor $f_{u p}$, that represents a correction factor used in the following calculation of real radiation, is given by

$$
f_{u p}=\frac{64-\delta_{u p_{A}}}{64}
$$

All calculations are made by assuming that the total sunlight reflected by the mirrors affects the absorber's pipe or secondary reflector. This is guaranteed by the large mirror's radius of curvature. In fact, due to high mirror's radius of curvature there are no reflected radiation losses.

The real solar radiation that affects the receptor is different from the theoretical one, because it takes into account all the losses and correction factors obtained in the previous subsection. The contribution that each mirror gives to the solar radiation that affects the receptor (in Watts $/ \mathrm{m}^{2}$ ) is computed according to the following equation:

$$
\operatorname{rad}_{\text {real }_{i}}=d r \cdot A_{i} \cdot \cos \kappa_{i} \cdot f_{u p} \cdot \zeta_{m} \cdot \zeta_{s r}
$$

where

- $d r$ is direct solar radiation $\left(\mathrm{W} / \mathrm{m}^{2}\right)$;

- $A_{i}$ is $i$-th row collectors area $\left(32 \mathrm{~m}^{2}\right)$;

- $\cos \kappa_{i}$ is optical losses factor for i-row;

- $f_{u p}$ is receptor unlit part factor;

- $\zeta_{m}$ is mirror nominal reflectivity $(0.92)$;

- $\zeta_{s r}$ is secondary reflector nominal reflectivity (0.77).

The total real solar radiation that affects the receptor is the sum of the real reflected solar radiation of all rows, multiplied by the total shadow factor $f_{t o t}$ :

$$
\operatorname{rad}_{\text {real }_{\text {tot }}}=\sum_{j=1}^{11} \operatorname{rad}_{\text {real }_{j}} \cdot f_{\text {tot }} \text {. }
$$

The theoretical solar radiation $\left(\mathrm{W} / \mathrm{m}^{2}\right)$ can be easily computed without taking into account the optical loss factor $\cos \kappa$ and the total shadow factor $f_{t o t}$, and hence

$$
\operatorname{rad}_{\text {theotot }}=d r \cdot A_{t o t} \cdot \zeta_{m} \cdot \zeta_{s r}
$$

where $A_{t o t}$ is the total reflective surface, equal to $352 \mathrm{~m}^{2}$.

\section{THERMAL MODEL}

The thermal model of the plant is focused on heat exchanges in the receptor. All geometrical and structural data (like length, exterior diameter, thickness) of absorber pipe, glass cover and secondary reflector are known as well as thermal parameters necessary to calculate heat flow, absorptivity and emissivity. In the thermal model, the quantities that have been considered as input are:

- Ambient temperature $\left({ }^{\circ} \mathrm{C}\right)$;

- Inlet water temperature $\left({ }^{\circ} \mathrm{C}\right)$;

- Water flow $\left(\mathrm{m}^{3} / \mathrm{s}\right)$;

- Real solar radiation, obtained with the optical model $\left(\mathrm{W} / \mathrm{m}^{2}\right)$.

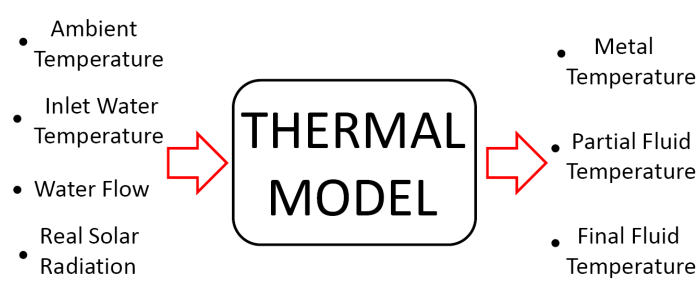

Fig. 4. Thermal model scheme.

The thermal model allows to know temperature distribution in the absorption pipe and in heat-transfer fluid (water) along the pipe at a given moment, as well as the temporary variation of the temperature at determined points of the pipe.

For the model the same hypotheses made in Camacho et al. [1997] have been adopted. Under these assumptions, by applying the conservation of the energy in the metal pipe of a length volume $d x$ over a time interval $d t$, the 
energy balance for each infinitesimal volume is obtained. Time variation of internal energy on each volume can be expressed as:

$$
\frac{\partial U}{\partial t}=\rho_{m} C_{m} A_{m} \frac{\partial T_{m}}{\partial t} \partial x
$$

where $C_{m}$ is the specific heat capacity of the metal, $A_{m}$ is the metal section area, $T_{m}$ is the temperature of the metal in Celsius and $\rho_{m}$ is the metal density.

This variation of internal energy is equals to the difference between the energy of solar radiation incident on absorber pipe and the sum of the energy ceded by the pipe to the ambient and the energy transferred to the fluid. The energy that affects the absorber pipe is the energy due to real solar radiation reflected by collectors. The expression for solar radiation energy is:

$$
E_{\text {rad }}=\omega_{r} \cdot B \cdot d x
$$

where $\omega_{r}$ is the real solar radiation and $B$ is the total collector aperture.

The energy transferred by absorber pipe to the ambient and the energy transferred to the fluid have a similar expression:

$$
\begin{gathered}
E_{a m b}=H_{l}\left(T_{m}-T_{a}\right) B d x \\
E_{f}=H_{t}\left(T_{m}-T_{f}\right) L d x
\end{gathered}
$$

where $T_{a}$ and $T_{f}$ are the temperatures of the ambient and of the fluid in Celsius degrees, $L$ is the inner diameter of absorber pipe and $H_{l}, H_{t}$ are the coefficient of global thermal losses and the coefficient of metal-fluid transmission, respectively, both expressed in $W / m^{2} C$. Therefore, the variation of internal energy is equal to:

$$
\begin{aligned}
\frac{\partial U}{\partial t} & =\omega_{r} \cdot B \cdot d x-H_{l}\left(T_{m}-T_{a}\right) \cdot B \cdot d x \\
& -H_{t}\left(T_{m}-T_{f}\right) \cdot L \cdot d x
\end{aligned}
$$

The global coefficient of thermal losses is considered per unit of mirror area, that is the power in Watts lost per mirror square meter and Celsius degree. The coefficient of metal-fluid transmission corresponds to the thermal power lost through the contact surface between fluid and metal.

The energy balance in the metal can thus be expressed as:

$$
\begin{aligned}
\rho_{m} C_{m} A_{m} \frac{\partial T_{m}}{\partial t} & =\omega_{r} B-H_{l} B\left(T_{m}-T_{a}\right) \\
& -L H_{t}\left(T_{m}-T_{f}\right) .
\end{aligned}
$$

In a similar manner, the first law of thermodynamics can be applied to the volume of fluid considered:

$$
\frac{\partial U}{\partial t}=H_{t} L\left(T_{m}-T_{f}\right) d x-\dot{m}\left(\bar{H}_{x+d x}-\bar{H}_{x}\right)
$$

where $\dot{m}$ is the infinitesimal fluid element mass variation, $H_{t}$ is the coefficient of metal-fluid transmission just described while $\left(\bar{H}_{x+d x}-\bar{H}_{x}\right)$ is the difference in enthalpy on the fluid element of base area $A_{f}$ and length $d x$. The enthalpy variation is equal to

$$
\frac{\partial \bar{H}}{\partial x} d x=C_{f} \frac{\partial T_{f}}{\partial x} d x
$$

under the assumption that specific heat capacity at constant pressure do not vary in the fluid element considered. Thus, internal energy variation in the infinitesimal element can be indicated as:

$$
\frac{\partial U}{\partial t}=H_{t} L\left(T_{m}-T_{f}\right) d x-\dot{q} \rho_{f} C_{f} \frac{\partial T_{f}}{\partial x} d x
$$

where $\dot{q}$ is the water flow in $m^{3} / s$. On the other hand, the internal energy variation in the fluid element can be expressed as a function of the fluid temperature:

$$
\frac{\partial U}{\partial t}=\rho_{f} C_{f} A_{f} d x \frac{\partial T_{f}}{\partial t}
$$

where $A_{f}$ is the fluid section area, $\left(A_{f} \cdot d x\right)$ is the fluid element volume, $\rho_{f}$ the fluid density and $C_{f}$ the fluid specific heat capacity. Using this relation, energy balance in the fluid element has the following expression:

$$
\rho_{f} C_{f} A_{f} \frac{\partial T_{f}}{\partial t}+\rho_{f} C_{f} \dot{q} \frac{\partial T_{f}}{\partial x}=H_{t} L\left(T_{m}-T_{f}\right) .
$$

The equations for the energy balance in the infinitesimal element are therefore the following, where the subscripts $m$ and $f$ are referred respectively to metal and fluid:

$$
\begin{aligned}
& \rho_{m} C_{m} A_{m} \frac{\partial T_{m}}{\partial t}=\omega_{r} B-H_{l} B\left(T_{m}-T_{a}\right)+ \\
&-H_{t} L\left(T_{m}-T_{f}\right) \\
& \rho_{f} C_{f} A_{f} \frac{\partial T_{f}}{\partial t}+\rho_{f} C_{f} \dot{q} \frac{\partial T_{f}}{\partial x}=H_{t} L\left(T_{m}-T_{f}\right) .
\end{aligned}
$$

These equations are only applicable to active zones, that is, the parts of absorber pipe that receive the beam radiation. The equations that describe the performance in a passive element are similar, except that solar energy input is nil. Thus, the model for the complete field is built by a series of active and passive elements

The equations derived can be solved using an iterative process with finite differences. The temperature of the fluid and of the absorber pipe are calculated for each time interval and for each element. For the plant considered, a value of 1 meter for $d x$ length and a time integration interval of 0.5 seconds have been chosen.

A two stage algorithm has been implemented to solve temperature equations. In the first stage the temperatures of the fluid and of the metal are calculated supposing that the fluid is in a steady state. In the second stage the fluid temperature is corrected in function of the net energy transported by the fluid.

$$
\begin{aligned}
1^{\text {st }} \text { Stage } & \\
T_{m}(n, k)= & T_{m}(n, k-1)+\frac{\Delta t}{\rho_{m} C_{m} A_{m}} \cdot \\
\cdot & \left(\omega_{r} B-H_{l} B\left(T_{m}(n, k-1)-T_{a}(k)\right)\right. \\
- & H_{t} L\left(T_{m}(n, k-1)-T_{f}(n, k-1)\right) \\
T_{f_{p}}(n, k)= & T_{f_{p}}(n, k-1)+ \\
& \frac{H_{t} L \Delta t}{\rho_{f} C_{f} A_{f}}\left(T_{m}(n, k-1)-T_{f}(n, k-1)\right) \\
2^{n d} \text { Stage } & \\
T_{f}(n, k)= & T_{f_{p}}(n, k)
\end{aligned}
$$




$$
\left.-\frac{\dot{q} \Delta t}{A_{f} \Delta x}\left(T_{f_{p}}(n, k)-T_{f_{p}}(n-1, k)\right)\right)
$$

In these difference equations, $T_{f}(n, k)$ and $T_{m}(n, k)$ are the temperatures of fluid and metal in the segment $n$ during the $k$-th time interval, while $T_{a}(k)$ is the ambient temperature during the $k$-th time interval. Moreover $T_{f_{p}}$ is the fluid partial temperature before the correction while $T_{f}$ is the final fluid temperature. Thus, the outputs of the model are:

- Metal temperature of each pipe segment $\left({ }^{o} \mathrm{C}\right)$;

- Partial fluid temperature of each pipe segment $\left({ }^{o} \mathrm{C}\right)$;

- Final fluid temperature of each pipe segment $\left({ }^{\circ} \mathrm{C}\right)$.

\subsection{Model Identification}

Although all variables are needed for a complete description of the process, the only output of the thermal model that can be used for a comparison to real data is the final temperature of the last segment of the absorber pipe. In fact there are only two temperature sensors, one at the beginning and one at the end of absorber pipe, and so is not possible to compare the temperatures of the model internal segments with real ones, neither the metal temperatures of the pipe segments can be compared to the real ones, because there are not sensors to monitor them.

All geometrical and structural data of the plant are known and thus the constant values of the model can be determined. The only magnitudes that are unknown are the global losses coefficient $H_{l}$ and the metal-fluid transmission coefficient $H_{t}$. These coefficients are typical of the particular process, and so are valid only for the dynamics of the plant considered. $H_{l}$ and $H_{t}$ have been expressed as polynomial functions of second degree of the temperature.

The different parameters of thermal model equations have been determined using real data from the plant. The best fit in the least-squares sense minimizes the sum of squared residuals, a residual being the difference between an observed value and the value provided by a model. In this case, the model developed is linear in the parameters and a numerical algorithm for linear model is used to solve it. The model can be expressed in matrix form as $y=A \theta+\epsilon$, where $\theta$ is the vector of parameters, $y$ is the vector of measurements, $A$ a suitable matrix, while $\epsilon$ is the vector of measurement errors.

\section{VALIDATION AND EXPERIMENTAL RESULTS}

The model has been validated with real data from the plant. Figures in this section shows the water output temperature given by the model and the real one for a clear day (Figure 5) and for a particular windy and cloudy day (Figure 6).

As it can be seen, the model prediction curve follows very well the real one in the clear day, while in the second case the presence of the wind and of banks of clouds changes the heat transmission condition, issues that are not taken into account in the model. In fact, the wind especially if strong, influences the thermal conductivity to the ambient, increasing the thermal losses. Moreover the presence of banks of clouds hinders further temperature increase and results in a series of peaks that are still followed well enough by the model with a maximum difference between real and model data of about $8^{\circ} \mathrm{C}$. Furthermore, direct solar radiation was estimated from the global radiation measurements and the estimation errors are bigger with clouds. In any case, the dynamic model developed reproduces the dynamic of the real plant with enough accuracy to be used for testing control strategies.

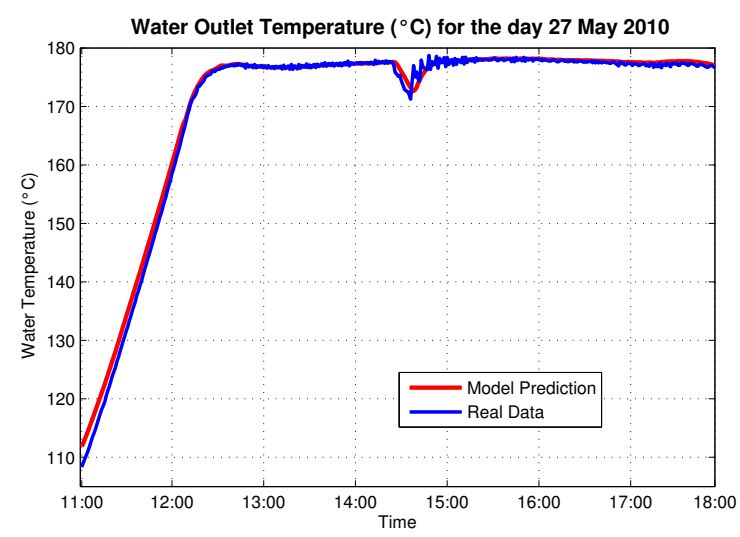

Fig. 5. Comparison between model predictions and real data of water outlet temperature for May 27, 2010.

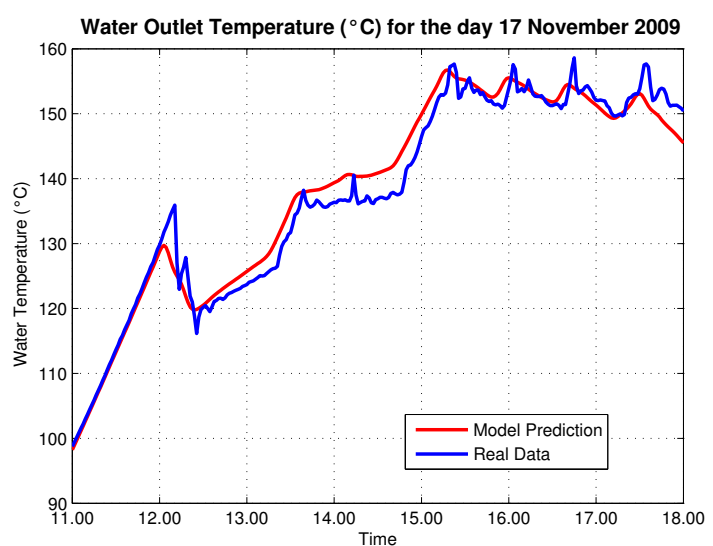

Fig. 6. Comparison between model predictions and real data of water outlet temperature for Nov.17, 2009.

\section{REFERENCES}

A. Larbi, M. Godin, J. Lucas (2000). Analysis of two models of (3D) Fresnel collectors operating in the fixedaperture mode with a tracking absorber. Solar Energy, 69, No. 1:1-14.

J. Faco and A.C. Oliveira (2009). Numerical simulation of a linear Fresnel solar collector concentrator. SET2009 - 8th International Conference on Sustainable Energy Technologies, Aachen (D).

E.F. Camacho, M. Berenguel and F.R. Rubio (1997). Advanced Control of Solar Plants. Springer.

M. Spoladore (Supervisors: E.F. Camacho and M.E. Valcher) (2010). Analysis and Modeling of a Thermosolar Plant. Automation Engineering Master Thesis.

P. Bermejo, F.J. Pino and F. Rosa (2010). Solar absorption cooling plant in Seville. Solar Energy, 84: 1503-1512. 